\title{
Improving Data Quality in Atom Probe Tomography
}

D.J. Larson ${ }^{1}$, T.J. Prosa ${ }^{1}$, D. Lawrence ${ }^{1}$, S.N. Strennen ${ }^{1}$, E. Oltman ${ }^{1}$, I. Martin ${ }^{1}$, D.A. Reinhard ${ }^{1}$, A. D. Giddings $^{1}$, D. Olson ${ }^{1}$, J.H. Bunton ${ }^{1}$, R.M Ulfig ${ }^{1}$, T.F. Kelly ${ }^{1}$, J. R. Goodwin ${ }^{2}$ and R.L. Martens ${ }^{2}$

${ }^{1}$ CAMECA Instruments Inc., 5500 Nobel Drive, Madison, WI 53711 USA

${ }^{2}$ Metallurgical and Materials Engineering, University of Alabama, Tuscaloosa, AL, 35487 USA

The electric fields used in atom probe tomography (APT) generate stresses normal to the specimen surface proportional to the electric field squared [1]. For example, typical APT evaporation fields of $30-60 \mathrm{~V} / \mathrm{nm}$ result in stresses of the order 1 tonne $/ \mathrm{mm}^{2}$ [2]. As such, specimen fracture has long been a serious problem for APT [3-6].

Figure 1 schematically illustrates several methods to improve APT specimen yield through decreasing stress by decreasing the evaporation field required during data collection: 1) decrease ion detection rate, 2) increase specimen base temperature, 3) use laser rather than voltage pulsing, and 4) increase laser pulse energy. The current work explores the use of thin coatings to modify the thermal and/or optical properties of 302 stainless steel, $\mathrm{SiN}$, or a $\mathrm{Si} / \mathrm{SiO} 2 / \mathrm{Si} / \mathrm{Ni}$ test structure (Fig. 2) in order to improve yield [7]. The APT data collection parameters were nominally: specimen temperature $30 \mathrm{~K}$, laser pulse energy $30 \mathrm{pJ}$ at $625 \mathrm{kHz}$, and a detection rate of one ion every 333-1000 pulses.

While method four above can have a positive effect on yield, extreme increases in laser energy are often limited in materials with poor thermal diffusivity due to a degradation in data quality (e.g., long "thermal tails" in the mass spectrum, non-uniform detector hit-maps, etc.) [8,9]. In order to investigate the effects of non-uniform detector hit-maps in a poor thermal diffusivity material, the data quality of 302SS was investigated at various laser energies. Median filtered detector density hit-maps at increasing energies from 10 to $200 \mathrm{pJ}$ per pulse are shown in Fig. 3a, which suggests an increasing trend in azimuthal asymmetry (note laser incidence is lower left). Fig. 3b presents the mass resolving power (MRP) at hundredth maximum, XY signal correlation from Fig. 3a (a value of zero means no correlation; a value $>0$ means correlation exists along $\mathrm{y}=\mathrm{x}$ direction), and spectral background as a function of laser energy. Generalized limits of low background and good (low) uniformity suggest a maximum laser energy of $\sim 75 \mathrm{pJ}$. SiN also has poor thermal diffusivity and shows substantially degraded MRP with increasing laser energy, shown in Fig. 4 for different specimen base temperatures. In both of these materials, higher energies may improve yield; however, data quality may be unacceptably degraded. In such cases, data collection may benefit by applying a coating of material to the specimen following sharpening (note single data point in Fig 4) [7]. In order to investigate this effect, we have developed the test structure shown in Fig. 2a, on which the effects of yield improvement method (1) above are shown very clearly in Fig. 5. Efforts to improve the yield in this test structure will be discussed in this presentation.

1. D. G. Brandon in, Field Ion Microscopy, eds. J. Hren, S. Ranganathan (Plenum, 1968) p.64.

2. E. W. Müller and T. T. Tsong, Prog. Surf. Sci. 4 (1973) p.1.

3. T. J. Wilkes et al., J. Phys. D: Appl Phys. 5 (1972) p.2226.

4. S. V. Zătsev, Tech. Phys. Lett. 30 (3) (2004) p.190.

5. I. M. Mikhailovskij et al., Ultramicroscopy 109 (209) p.480.

6. S. Kölling and W. Vandervorst, Ultramicroscopy 109 (2009) p.486. 
7. D. J. Larson et al., Microsc. Microanal. 19(S2) (2013) p.994.

8. J. H. Bunton et al., Microsc. Microanal. 13 (2007) p.418.

9. B. Gault et al., Ultramicroscopy 110 (2010) p.1215.

10. G. L. Kellogg, J. Appl. Phys. 53(9) (1982) p.6383.

1

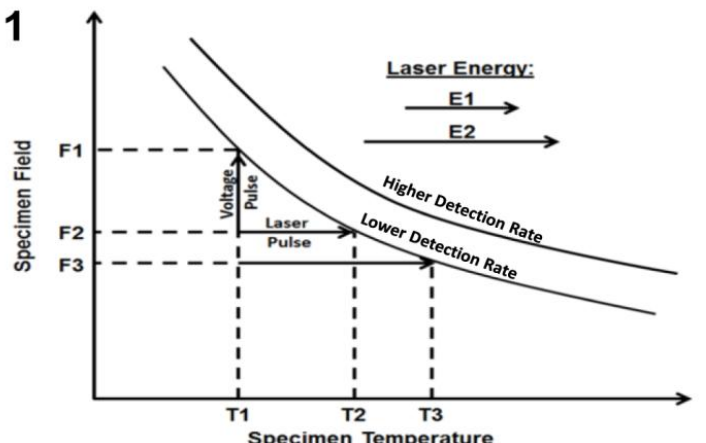

$3 a$
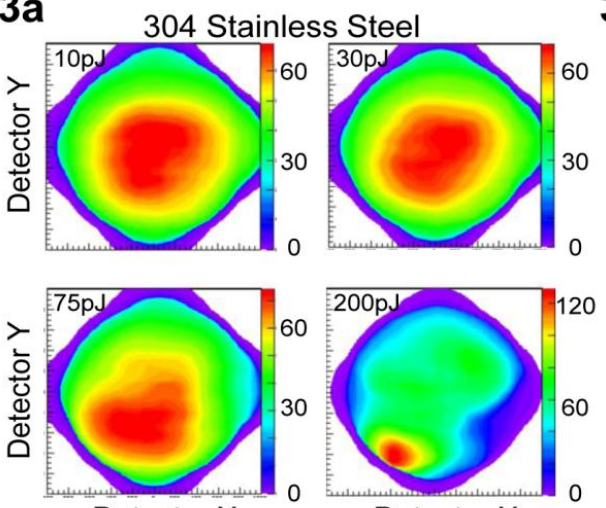

Detector X

4

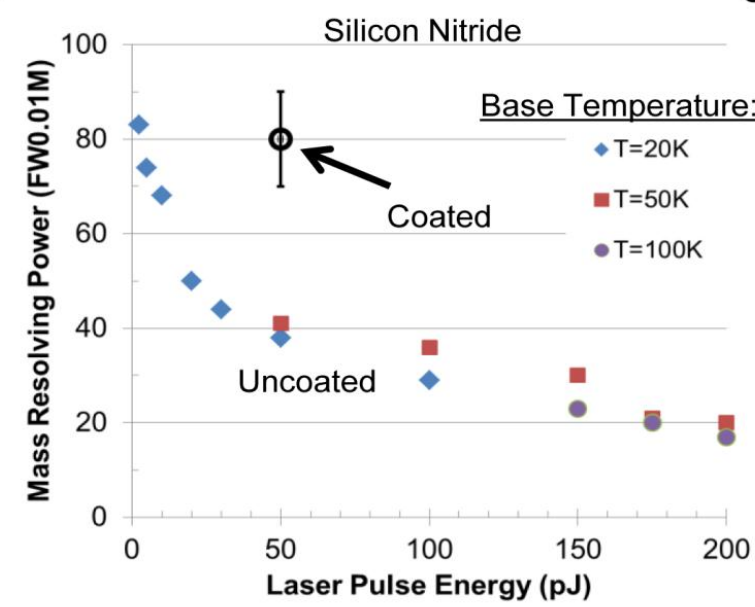

$3 b$
$2 a$
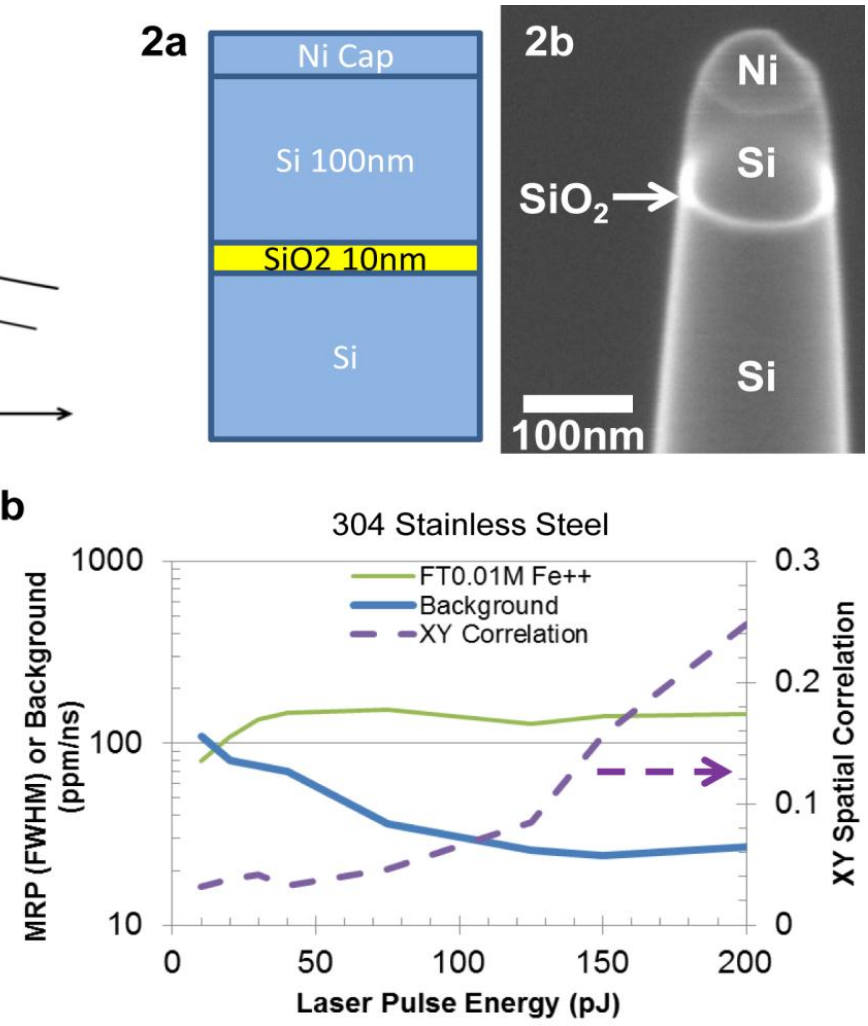

5

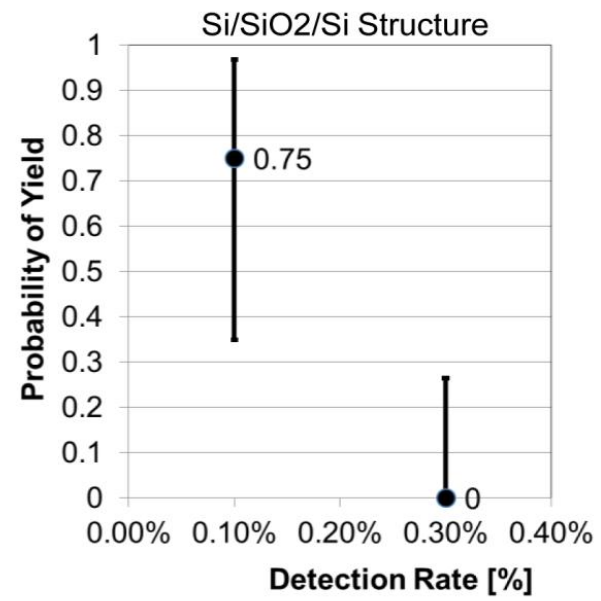

Fig. 1. Schematic diagram of evaporation field as a function of temperature.

Fig. 2. Oxide layer test structure used to measure yield. (a) Schematic and (b) SEM image of tip.

Fig. 3. (a) Detector density hit-maps for laser energies of 10, 30, 75, and $200 \mathrm{pJ}$, and (b) variation in FW0.01M mass resolving power, background, and hit-map XY correlation with laser energy.

Fig. 4. SiN mass resolution with laser energy for several different specimen base temperatures.

Fig. 5. Yield probability to analyze through entire structure in Fig 2a as a function of detection rate. 